\title{
1.3 Influence of hyperuricemia on changes of cardiac markers, functional and coronary reserves in patients with stable angina pectoris
}

Analysis of the literature shows the relationship between hyperuricemia and cardiovascular disease. Epidemiological and experimental studies over 10 years have shown that hyperuricemia is not only a risk factor for gout and nephrolithiasis, but is often accompanied by hypertension, type 2 diabetes, obesity, metabolic syndrome, chronic kidney disease and cardiovascular disease. In addition, comorbid pathology, such as renal failure or insulin resistance, leads to increased levels of uric acid in the serum, thereby contributing to the development of gout [23, 31]. It is recognized that gout forms a group with an increased risk of cardiovascular disease [27], in turn, atherosclerosis induces an increase of uric acid, which leads to an unfavorable prognosis in patients with acute heart failure.

As mentioned [25], the levels of C-reactive protein and N-terminal pro B-type natriuretic peptide, associated with an increase of serum uric acid $(1 \mathrm{mg} / \mathrm{dL}$, are independent indicators of 1-year mortality. Hyperuricemia increases the risk of hypertension (2-3 times in 5-7 years), renal damage (5 times in 2 years at plasma uric acid levels $>330 \mu \mathrm{mol} / 1$ ), progression of IgA nephropathy, associated with obstructive pulmonary syndrome sleep apnea, vascular diseases (carotid, peripheral and coronary arteries), stroke and vascular dementia, preeclampsia, inflammation, endothelial dysfunction, oxidative stress, sex [27, 28, 29]. Thus, the study of the mechanisms of interdependence of these indicators is relevant against the background of hyperuricemia and coronary heart disease.

We examined 120 patients with an objectified diagnosis of stable angina depending on the distribution of uric acid levels and manifestations of hyperuricemia without its symptoms (34 patients, $28.33 \%$ of cases) and with elevated uric acid levels (86 people, $71.67 \%$ of cases).

At the beginning of inpatient treatment [21] and after 6 months at the outpatient stage, patients were examined for uric acid, $\mathrm{N}$-terminal pro B-type natriuretic peptide and C-reactive protein, with the evaluation of echocardiographic results in comparing 
changes in left ventricular ejection fraction and the achieved threshold load at Bicycle ergometer test.

A study of the set of detection of correlation between the studied values with the estimation of correlation coefficients, two-dimensional descriptive statistics measuring the total variability of two or more variables represented by correlation coefficients, including Pearson's linear correlation coefficient, subsequent construction of sets of equations.

In the group of hyperuricemia more often diagnosed with stable angina III functional class $(87.21 \pm 3.60$ against $41.18 \pm 8.44 \%$ of cases of stable angina III functional class, $\mathrm{p}<0.001$ ), as well as the probability of a history of Q-Myocardial infarction, $60 \pm 6.81$ vs. $32.84 \pm 8.05 \%$ of cases, $p<0.05$ ), and when the uric acid level exceeds $467.9 \mu \mathrm{mol} / 1$, the left ventricular ejection fraction probably decreased (51.17 \pm 1.37 vs. $55.77 \pm 0.73 \%, p<0.01$ ), which was also realized by Bicycle ergometer test tests with probably lower threshold load values $(53.65 \pm 2.89 \mathrm{~W}$ in the hyperuricemia group against $72.74 \pm 5.18 \mathrm{~W}$ in the group with normal uric acid levels, $\mathrm{p}<0,01$ ) and was accompanied by an excess of uric acid levels above $467.9 \mu \mathrm{mol} / 1$, the predominance of C-reactive protein levels $(12.07 \pm 0.97$ vs. $5.37 \pm 0.60 \mathrm{mg} / 1, \mathrm{p}$ $<0.001)$ and N-terminal pro B-type natriuretic peptide $(413.44 \pm 63.14$ vs. $206.42 \pm$ $31.43 \mathrm{pg} / \mathrm{ml}, \mathrm{p}<0.01)$, as noted in previous publications $[22,30]$.

The following analysis is performed, as a separate case of stochastic connection, the study of correlation, in which the change in the mean value of the effective (changing under the influence of factor features) due to changes in factor (under their influence change other, dependent on them) features with model construction pair regression in the form of the equation of the relationship of two indicators $\mathrm{Y}$ (feature) and $\mathrm{X}$ (factor) to determine the effect of changes in one indicator on another, when the equation of the pairwise regression model is presented as $\mathrm{Y}=\mathrm{f}(\mathrm{X})$, where $\mathrm{Y}$ is the dependent indicator (effective sign), $\mathrm{X}$ is an independent (explanatory) factor.

The estimated regression equation (based on sample survey data at first entry to compare uric acid $(\mathrm{Y})$ and $\mathrm{N}$-terminal pro $\mathrm{B}$-type natriuretic peptide $(\mathrm{X})$ ) will be $\mathrm{Y}=$ $0.6501 * \mathrm{X}-38.0834$, correlation according to the Chaddock's scale between sign Y 
factor $X$ is noticeable and direct $\left(r_{X Y}=0.552\right)$. Since $\left|t_{\text {observ }}\right|>t_{c r i t}-$ we reject the hypothesis of equality 0 of the correlation coefficient, and the correlation coefficient itself is statistically significant, the study of the F-criterion (R. Fisher) shows that since the actual value of $F>F_{\text {table, }}$, the coefficient of determination is also statistically significant and regression is statistically reliable.

The correlation between uric acid $(\mathrm{Y})$ and $\mathrm{C}$-reactive protein level $(\mathrm{X})$ is described by the equation $\mathrm{Y}=0.02142 * \mathrm{X}-0.26$, is high and direct $(\mathrm{rXY}=0.701)$, according to the F-test is statistically reliable. In the equation with the estimate of the ratio of uric acid $(\mathrm{Y})$ and $\mathrm{PV} L V(\mathrm{X})$ by the formula $\mathrm{Y}=0.0134 * \mathrm{X}+45.0824$, but the relationship is weak $\left(r_{X Y}=0.224\right)$ and statistically unreliable by the F-test. The relationship between uric acid (Y) and threshold load (X) according to the equation $\mathrm{Y}$ $=-0.03104 * \mathrm{X}+41.0479$ and according to the F-test is statistically unreliable.

Thus, the comparison of the correlation of two indicators, one of which is effective, which changes under the influence of factor traits and is actually uric acid (Y) shows a high and direct relationship between assessment factors for C-reactive protein, noticeable and direct - for N-terminal pro B-type natriuretic peptide, both according to the F-criterion are statistically reliable, in contrast to the weak and statistically unreliable - for left ventricular ejection fraction and threshold load.

The next task of the study is to build multiple regression with the creation of a model with a large number of factors, determining the impact of each of them separately, as well as their combined impact on the simulated indicator, because multiple regression can analyze the relationship between several independent variables (predictors) and the dependent variable (Y), which in its own work is the content of uric acid in the first receipt, while the influencing factors, where $\mathrm{X}=\mathrm{X}\left(\mathrm{X}_{1}, \mathrm{X}_{2}, \ldots, \mathrm{X}_{\mathrm{m}}\right)$ - a vector of independent (explanatory) variables, it is proposed to evaluate the indicators: $\mathrm{X}_{1}$ - N-terminal pro B-type natriuretic peptide at the first admission and $\mathrm{X}_{2}$ - its content at the second, $\mathrm{X}_{3}-\mathrm{C}$-reactive protein at the first admission and $\mathrm{X}_{4}$ - its content at the second, $\mathrm{X}_{5}-$ left ventricular ejection fraction at the first admission and $\mathrm{X}_{6}$ - its level at repeated, $\mathrm{X}_{7}$ - threshold load at the first receipt and $\mathrm{X}_{8}$ - its achievement at repeated inspection in 6 months. The multiple regression equation is presented as: 


$$
\mathrm{Y}=-12.0028+0.3893 * \mathrm{X}_{1}-0.08975 * \mathrm{X}_{2}+13.4637 * \mathrm{X}_{3}-3.9902 * \mathrm{X}_{4}+
$$
$8.8012 * \mathrm{X}_{5}-2.0567 * \mathrm{X}_{6}-6.233 * \mathrm{X}_{7}+3.594 * \mathrm{X}_{8}$

The observed values of $t$-statistics for ryX1-8 are calculated and defined table, $t_{c r i t}$ $(n-m-1 ; \alpha / 2)$. Since $t_{\text {observ }}>t_{\text {crit }}$, we reject the hypothesis of equality 0 of the correlation coefficient and the correlation coefficient is statistically significant, which is registered for factors $\mathrm{X}_{1}\left(\mathrm{~N}\right.$-terminal pro B-type natriuretic peptide $\left.{ }_{1}\right) \mathrm{t}_{\mathrm{observ}}=4.5 ; \mathrm{t}_{\text {crit }}(\mathrm{n}-\mathrm{m}-1 ; \alpha /$ $2)=(46 ; 0.025)=2.009 ; \mathrm{X}_{3}\left(\mathrm{C}\right.$-reactive protein $\left.{ }_{1}\right)$ statistically significant, $\mathrm{t}_{\text {observ }}=4.5$; $\mathrm{X}_{6}$ (left ventricular ejection fraction ${ }_{2}$ ) statistically significant, $\mathrm{t}_{\mathrm{observ}}=3.52$.

Interestingly, no significant dependence of uric acid content on the indicators $\mathrm{X}_{5}$

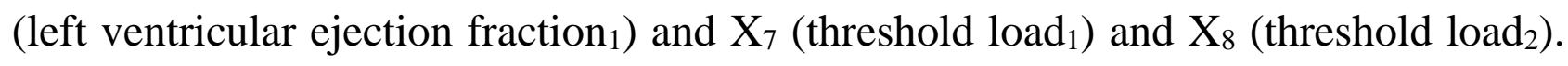
At the same time, the initial level of uric acid was not expected to be affected by the factor of the second intake $\mathrm{X}_{2}$ (N-terminal pro B-type natriuretic peptide $\left.\mathrm{N}_{2}\right), \mathrm{X}_{4}(\mathrm{C}$ reactive $\left.\operatorname{protein}_{2}\right)$. Checking the overall quality of the multiple regression equation using F-statistics (Fisher's test) with the assessment of the relationship for the predominance of the actual value of $\mathrm{F}>\mathrm{F}_{\mathrm{kp}}$, and therefore in this case the coefficient of determination was statistically significant and the regression equation was statistically reliable.

It was found that factor $\mathrm{X}_{1}\left(\mathrm{~N}\right.$-terminal pro B-type natriuretic peptide $\left.{ }_{1}\right) \mathrm{F}_{\mathrm{X} 1}$ (50.094)> 2.25, therefore, factor $X_{1}$ should be included in the model after the introduction of factors $X_{j}$, with a similar dependence for $X_{3}\left(C\right.$-reactive protein $\left.{ }_{1}\right) F_{X 3}$ (55.276)> 2.25; $\mathrm{X}_{4}(\mathrm{C}$-reactive protein 2$) \mathrm{F}_{\mathrm{X} 4}(6.848)>2.25 ; \mathrm{X}_{5}$ (left ventricular ejection fraction $\left._{1}\right) F_{X 5}(22.6)>2.25$ and $X_{7}\left(\right.$ threshold load $\left._{1}\right) F_{X 7}$ (37.024)> 2.25.

Since the density of the combined influence of factors on the result allows to determine the multiple correlation index - the study was subjected to a multiple correlation coefficient through a matrix of paired correlation coefficients, it was determined that when approaching $\mathrm{R}$ to 1 (in our own study $\mathrm{R}=0,8933$ ), the regression equation better describes actual data affect the result.

Finally: defined as the correlation coefficient between variables belonging to the ordinal scale with a score for variable scale variables, parametric Pearson correlation coefficient - r-Pearson - a correlation measure for two continuous (metric variables) 
measured on the same sample, the score relationships between two series of indicators in the quantitative scale) [20].

It was found that the level of uric acid in the first examination shows a direct moderate relationship with $\mathrm{N}$-terminal pro B-type natriuretic peptide ${ }_{1}$ (r-Pearson 0,363, $\mathrm{p}<0,01$ ), a direct moderate relationship with C-reactive $\operatorname{protein}_{1}$ (r-Pearson $0,454, p<0$ , 01), inverse moderate with left ventricular ejection fraction ${ }_{1}$ (r-Pearson $-0,351, p$ $<0,01)$, acquires a direct high connection with $\mathrm{C}$-reactive protein $_{2}$ of the second admission ( $\mathrm{r}$-Pearson $0,765, \mathrm{p}<0,01$ ), direct moderate communication tongue with $\mathrm{N}$ terminal pro B-type natriuretic peptide 2 ( $\mathrm{r}$-Pearson $0.438, \mathrm{p}<0.01$ ), inverse moderate with left ventricular ejection fraction $2(r-P e a r s o n-0.351, p<0.01)$.

In turn, $\mathrm{N}$-terminal pro B-type natriuretic peptide of the first admission showed a direct appreciable connection with C-reactive $\operatorname{protein}_{1}$ (r-Pearson 0,533, p <0,01), inverse moderate with left ventricular ejection fraction $_{1}$ ( $r$-Pearson $-0,561, p<0,01$ ), further N-terminal pro B-type natriuretic peptide of the second admission showed an even more pronounced direct visible relationship with C-reactive protein 2 (r-Pearson $0.619, \mathrm{p}<0.01)$ and the reverse noticeable with left ventricular ejection fraction 2 ( $r$ Pearson -0.611, $\mathrm{p}<0.01)$.

The relationship between C-reactive protein and left ventricular ejection fraction was inverse moderate in the first ( $\mathrm{r}$-Pearson $-0.340, \mathrm{p}<0.01)$ and inverse moderate in the second ( $\mathrm{r}$-Pearson $-0.504, \mathrm{p}<0.01$ ) surveys. Noteworthy is the lack of Pearson correlation in the assessment of the achieved load in Bicycle ergometer test except for the weak feedback in the second survey with $\mathrm{N}$-terminal pro B-type natriuretic peptide 2 (r-Pearson -0.319, $\mathrm{p}<0.05)$.

Therefore, the study of changes in the Pearson correlation coefficient depending on the levels of uric acid, N-terminal pro B-type natriuretic peptide and C-reactive protein showed a predominantly direct moderate relationship at admission, which reached a noticeable and even high relationship in the second survey, with the inverse moderate for left ventricular ejection fraction, in contrast from the achieved load at Bicycle ergometer test. 
In conclusion, the following can be noted:

1. Hyperuricemia causes coronary reserve limitation with increasing functional class of angina and reduction of threshold load, decreased left ventricular ejection fraction, increased levels of C-reactive protein, N-terminal pro B-type natriuretic peptide, with the reverse reaction of total testosterone in general.

2. The estimated regression equation, according to the sample survey data, at the first admission to compare uric acid with the N-terminal pro B-type natriuretic peptide indicates a noticeable and direct connection, C-reactive protein - high and direct, according to the F-test is statistically reliable, in contrast to the weak and statistically unreliable - for the fraction of left ventricular ejection according to echocardiography and threshold load during bicycle ergometer test.

3. Construction of multiple regression equation with dependent variable uric acid content and influencing factors - C-reactive protein, N-terminal pro B-type natriuretic peptide, left ventricular ejection fraction, threshold load is statistically reliable with a high coefficient of multiple correlation $(r=0,89)$.

4. The Pearson correlation coefficient depending on the levels of uric acid, $\mathrm{N}$-terminal pro B-type natriuretic peptide and $\mathrm{C}$-reactive protein indicates a predominantly direct moderate binding on admission, with a noticeable and even high binding on re-examination, with a moderate feedback for left ventricular ejection fraction, in contrast to the achieved load during bicycle ergometer test. 